\title{
An Intelligent Fault Diagnosis Model Based on FastDTW for Railway Turnout
}

\author{
$1^{\text {st }}$ JI Wen-Jiang ${ }^{12}, 2^{\text {nd }}$ ZUO Yuan ${ }^{1}, 3^{\text {rd }}$ HEI XIN-Hong ${ }^{1}, 4^{\text {th }}$ FEI Rong ${ }^{1}$ \\ \{wjj@xaut.edu.cn,759666216@qq.com, heixinghong@xaut.edu.cn, annyfei@xaut.edu.cn\} \\ School of Computer Science and Engineering, Xi' an University of Technology, Xi' an, China ${ }^{1}$, \\ State Key Laboratory of Rail Transit Engineering Informatization FSDI, Xi'an, China ${ }^{2}$
}

\begin{abstract}
The turnout handles the direction of the train which is one of the key equipment in the railway transportation system. In this paper, by using real action current data obtained from switch machine model No.ZD7, a turnout fault diagnosis model based on the FastDTW pattern recognition algorithm was proposed. Firstly, the original current curve was segmented relate to the features of them. Then the warping path distance between the standard sample and the tested current curve was obtained according to FastDTW algorithm. Finally a dynamic optimized threshold was used to confirm whether there is a fault happened in the turnout. According to the experiment results, the proposed diagnose model without the prior knowledge of fault samples can works well both with single and double acting type turnout machines, owning to the following elements: the diagnose accuracy can be more than $96 \%$, the time-cost can be improved more than 5 times compared with traditional DTW based algorithms.
\end{abstract}

Keywords: turnout; switch machine; fault diagnosis; FastDTW

\section{Introduction}

Railway turnout system is an important part of railway infrastructure and a frequent failure part in rail transit. Its health directly affects the safety of the railway system. At present, enhancing the safety of turnouts mainly depends on setting the alarm threshold of the signal curve, Periodic inspection of system logs by professional and technical personnel to determine the health status of turnouts. However, this method is inefficient, and the phenomenon of underreporting and misreporting is common, which cannot meet the requirements of railway safety guarantee.

With the continuous development of artificial intelligence and big data technologies, various intelligent fault diagnosis methods for turnouts are gradually being widely studied. In essence, fault diagnosis is a pattern recognition problem. As a powerful pattern recognition technology, artificial intelligence has been widely used in turnout fault problems. For example, Bayesian networks ${ }^{[1]}, \mathrm{SVM}^{[2]}, \mathrm{HMM}^{[3]}$, etc. However, most of the existing research methods of turnout fault diagnosis focus on the signal data of single action turnout. There are few related researches on the fault diagnosis of double action turnout. In view of the above reasons, based on the NO.ZD7 turnout switch operating current data, this paper proposes a FastDTWbased turnout fault diagnosis method by analyzing the warp path distance between the switchpoint current and the standard template data to be diagnosed. Experimental data shows that the method proposed in this paper does not require a large amount of prior fault data, and 
the algorithm accuracy rate is more than $96 \%$, and the time performance is improved by more than 5 times compared with the traditional DTW algorithm. It is Suitable for new train control systems with higher accuracy and real-time requirements.

\section{Methodology}

Dynamic Time Warping(DTW) algorithm is used to calculate the distance similarity of any two time series, and it is not necessary that the two time series have the same length ${ }^{[4]}$. It uses the method of dynamic programming to find the warp path, and its time complexity is $\mathrm{O}(\mathrm{m} \times \mathrm{n})$. When the time series is long, the efficiency of the algorithm is very low. FastDTW ${ }^{[5]}$ algorithm accelerates DTW algorithm by limiting the scope of path search and data abstraction. It is an improved algorithm on the time complexity of DTW algorithm. The algorithm can find an approximate optimal warp path between two time series, and the time consumption increases linearly with the increase of input time series, and its time complexity is about $\mathrm{O}(\mathrm{n})$. FastDTW algorithm has three main processes: coarsening, projection and refinement. Figure 1 shows the speedup of fastdtw

(1) Coarsening: Shrink the original time series, using half of the original time series sampling points to represent the original time series, and the value of each sampling point of the shrunk time series is the mean value of two adjacent sampling points of the original time series. As shown in Figure 1, the distance matrix under the 1/1 granularity is the original distance matrix, and the $1 / 2,1 / 4$ and $1 / 8$ granularity are the matrices during the three coarsening process of the original time series.

(2) Projection: DTW algorithm is run on the distance matrix of lower resolution. As shown in Figure 1, the black line segment is the warp path found by running DTW algorithm on this granularity.

(3) Refinement: After finding the warp path on the lower resolution matrix, the mesh that the warp path passes through is mapped to the higher resolution matrix.

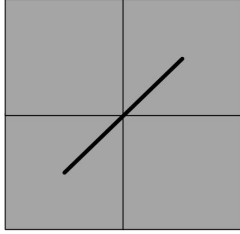

$1 / 8$

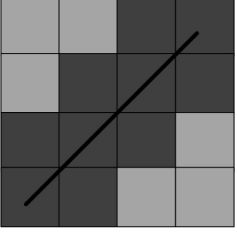

$1 / 4$

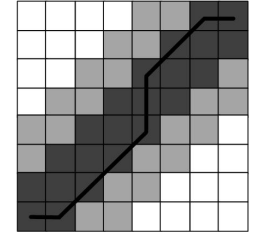

$1 / 2$

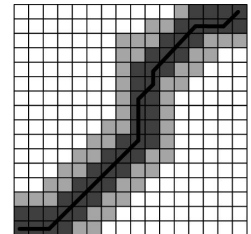

$1 / 1$

Fig. 1. The acceleration process of FastDTW.

The core idea of FastDTW algorithm is to speed up DTW by limiting the search scope and data abstraction, that is, to search only the grid in the projection distortion path (the deepest meshes in Figure 1). However, the optimal warp path may not be included in the projected warp path, so the FastDTW algorithm adds a radius parameter, it is allowed to search the radius meshes outside the projection distortion path mesh, that is, the shading lighter mesh in Figure 1 (radius in Figure 1 is 1). The larger the radius, the more accurate the warp path will be. If the radius parameter is set to the same length as the input time series, the FastDTW algorithm and DTW algorithm have the same efficiency. 


\section{Turnout fault diagnosis model based on FastDTW}

\subsection{Characteristic analysis of turnout action current curve}

With the continuous development of railway signal system, sensors are often equipped in key equipment of the system, such as switch machine, to monitor the running signal data of the equipment ${ }^{[6]}$. When the switch fails, its abnormal action process will be fed back to the switch machine, resulting in abnormal monitoring signal data. Therefore, the current curve of switch machine monitored in the process of switch action can reflect the health status of the switch. In this paper, the action current curve of the turnout equipment under the condition of operation at the time of delivery is taken as the standard template. If the difference between the action current curve to be measured and the action current curve of the template is large, it indicates that the turnout is likely to have faults in this action, so that the fault diagnosis of the turnout can be realized.

On the other hand, with the development of China's rail transit system, there are many types of turnout equipment, such as single acting, double acting and multi acting. The action signal data of double action and multi action turnout is more complex than that of single action. Figure 2 shows the normal current curve of single action turnout and double action turnout.

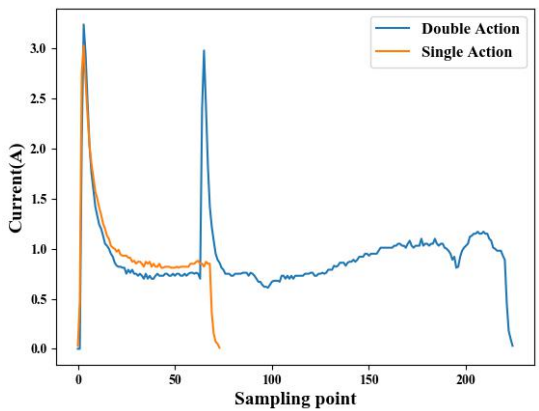

Fig. 2. Current curve of different types of turnout.

\subsection{Fault Diagnosis Based on FastDTW}

The warp path distance between the current curve to be diagnosed and the current curve of the template can be obtained by FastDTW algorithm. The warp path distance represents the difference between the curve to be diagnosed and the template curve. The smaller the distance of the warp path, the higher the similarity between the two curves, and the larger the distance, the lower the similarity between the two curves. Therefore, a threshold for the distance of a warp path can be set to determine the health status of the turnout. In addition, the turnout equipment has different operating conditions and different service lives, which results in different optimal diagnostic thresholds for different turnouts. Therefore, the setting of the optimal threshold needs to be continuously adjusted as the equipment changes to achieve a dynamic threshold.

Due to the varying length of each switch's action conversion process, the actual current data sampling lengths of the multiple monitoring switches are different, which leads to the difference in the distance of the warp path obtained by the FastDTW algorithm for different turnouts of the same model even if no fault occurs But relatively large, as a result, the effectiveness of the threshold is reduced and the difficulty of determining the threshold is increased. In this paper, in order to properly eliminate the influence of the difference in the 
length of the input data, the mean value of the warp path distance is processed, and the final warp path distance is calculated by FastDTW to divide the warp path distance into the curve length to be diagnosed.

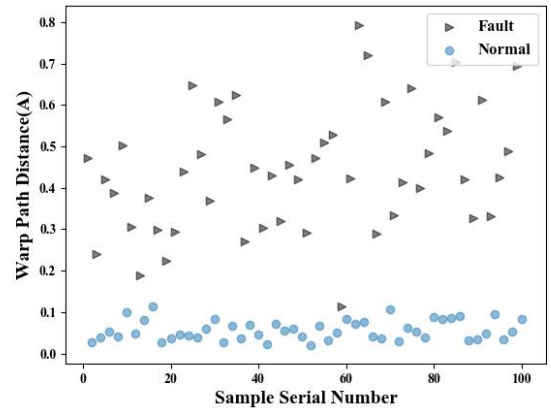

(a). Single action.

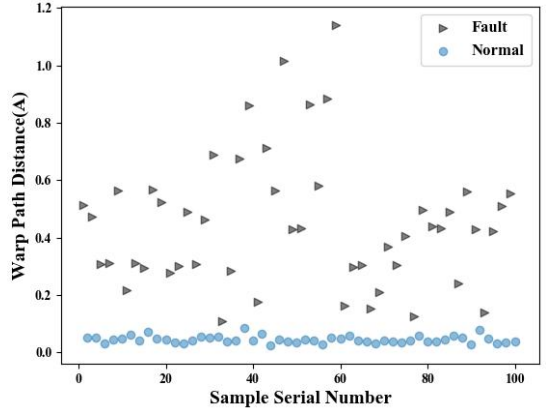

(b). Double action.

Fig. 3. The warp path distribution of different types of turnouts.

For how to confirm the fault judgment threshold, the warp path distances of normal current curve, fault current curve and its template current curve of different types of turnouts are analyzed in this paper. As shown in figure 3, figure 3(a) and figure 3(b) are respectively the warp path distance distribution diagrams of the current curve of the single action type turnout and the double action type turnout and its template current curve. It is not difficult to see from the figure that the distance between the normal curve of the single action turnout and the double action turnout and its template curve is mainly concentrated between $0.0 \mathrm{~A} \sim 0.1 \mathrm{~A}$. The distance between the fault curve and the template curve of the warp path exceeds this value, and the distribution is relatively discrete. Therefore, a threshold of warp path distance can be set to determine the health status of turnout.

\section{Model performance analysis}

In order to test the performance of the proposed model, based on the actual monitoring data of single-month switch machine operating current signals recorded by a railway bureau in China, experiments were performed on single action and double action turnouts. Among them, 200 pieces of data are used to determine the dynamic threshold, 120 pieces of data are used to test the proposed method (single action and double action ratio is 1:1). At the same time, a comparative experiment with DTW(ITDM-BD) and Frechet Distance ${ }^{[7]}$ is added

\subsection{Model accuracy analysis}

\subsubsection{Determination of the dynamic threshold}

In this section, we randomly trained 200 turnout current data according to the proposed model and Fresch distance-based turnout diagnosis method to obtain the optimal threshold of the training data set. It is divided into two types of threshold determination experiments: single action type and double action type. The experimental results are as follows..

Figure 4 shows the results of single and double action turnout fault diagnosis F1-Score for ITBM-BD, ITBM-BF and Frechet Distance. We choose the highest F1-score threshold as the best threshold, then the optimal thresholds of three models for single action turnout are $0.11 \mathrm{~A}$, 
$0.11 \mathrm{~A}$ and $2.1 \mathrm{~A}$ respectively and the best thresholds of double action turnout are $0.09 \mathrm{~A}, 0.09 \mathrm{~A}$ and $2.3 \mathrm{~A}$ respectively.

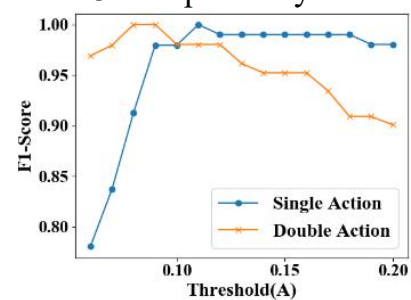

(a). ITDM-BD

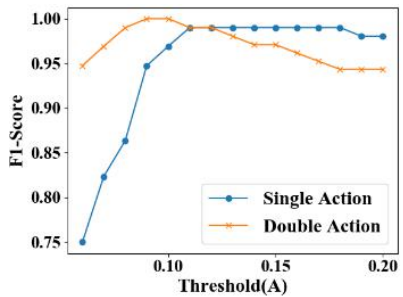

(b). ITDM-BF.

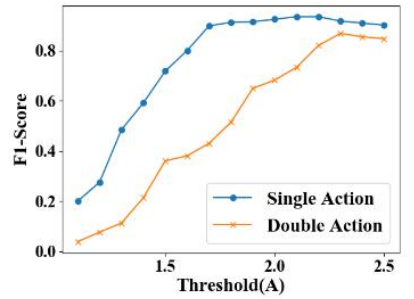

(c). Frechet Distance.

Fig. 4. F1-score comparison of three methods.

\subsubsection{Model accuracy test}

Based on the best thresholds determined in section 4.1.1, the model proposed in this paper is used to test the accuracy of the test set composed of 120 randomly selected data sets. The test results are shown in Table 1.

Table 1. Model accuracy test results.

\begin{tabular}{ccc}
\hline Model name & Turnout type & F1-Score \\
\hline \multirow{2}{*}{ ITDM-BD } & Single Action & $98.3 \%$ \\
& Double Action & $100 \%$ \\
\multirow{2}{*}{ ITDM-BF } & Single Action & $96.6 \%$ \\
& Double Action & $100 \%$ \\
Frechet Distance & Single Action & $88.7 \%$ \\
& Double Action & $84.3 \% \%$
\end{tabular}

As can be seen from Table 1, both ITDM-BD and ITDM-BF reach a higher F1-Score. Combining the F1-Score variation ranges of the two under different thresholds in Figure 4, it is not difficult to see that the two have little difference in accuracy performance, and both can meet actual application requirements. And both models are better than Frechet Distance based diagnosis model.

\subsection{Model time performance analysis}

In this section, 150 pieces of real data are divided into 5 test sets of different sizes, and the running time efficiency of ITDM-BD and ITDM-BF is tested respectively.

Table 2. Model run time.

\begin{tabular}{|c|c|c|c|c|c|c|}
\hline \multirow{2}{*}{ Model } & \multirow{2}{*}{ Turnout Type } & \multicolumn{5}{|c|}{ Input dataset size } \\
\hline & & 30 & 60 & 90 & 120 & 150 \\
\hline \multirow{2}{*}{ ITDM-BD } & Single & $14.11 \mathrm{~s}$ & $24.12 \mathrm{~s}$ & $34.91 \mathrm{~s}$ & $45.27 \mathrm{~s}$ & $63.93 \mathrm{~s}$ \\
\hline & Double & $25.42 \mathrm{~s}$ & $45.73 \mathrm{~s}$ & $65.67 \mathrm{~s}$ & $98.13 \mathrm{~s}$ & $120.96 \mathrm{~s}$ \\
\hline \multirow{2}{*}{ ITDM-BF } & Single & $2.09 \mathrm{~s}$ & $3.79 \mathrm{~s}$ & $5.13 \mathrm{~s}$ & $7.29 \mathrm{~s}$ & $9.67 \mathrm{~s}$ \\
\hline & Double & $3.60 \mathrm{~s}$ & $6.48 \mathrm{~s}$ & $9.31 \mathrm{~s}$ & $12.85 \mathrm{~s}$ & $16.82 \mathrm{~s}$ \\
\hline
\end{tabular}


As can be seen from Table 2, because ITDM-BF uses the FastDTW algorithm to calculate the warp path distance, its speed performance is significantly better than that of ITDM-BD, and the algorithm time efficiency is improved by at least 5 times.

\section{Conclusion}

For the fault diagnosis of turnout, this paper takes the current data of the switch machine monitored during the turnout operation as input, and obtains the current health status of the turnout to be diagnosed through artificial intelligence algorithms such as segmentation, pattern recognition and threshold determination. Experimental results show that ITDM-BD is not significantly different from ITDM-BF in accuracy performance, but the latter is significantly better than the former in terms of time efficiency. Therefore, ITDM-BF is more suitable for new train control systems with high accuracy and high real-time performance. In addition, without a large number of fault sample data, and for single- and double-acting turnout equipment, the model proposed in this paper can still efficiently and correctly diagnose turnout fault conditions, indicating that ITDM-BF has a wider scope of application. As the sample data is too small, it is necessary to verify whether the model in this paper is satisfied with the hyperactive turnout equipment, and the determination of the specific failure type of the turnout is still a future research direction.

\section{References}

[1] Jin W, Shi Z, Siegel D, et al. Development and evaluation of health monitoring techniques for railway point machines[C]. 2015 IEEE Conference on Prognostics and Health Management (PHM), 2015.

[2] Dong W, Liu M M, Wang L S, et al. Fault Diagnosis for Railway Turnout Control Circuit Based on Group Decision Making[J]. Acta Automatica Sinica, 2018, 44(6):47-56.

[3] Xu Q Y, Liu Z T, Zhao H. Method of Turnout Fault Diagnosis Based on Hidden Markov Model[J]. Journal of the China Railway Society, 2018, 250(08):102-110.

[4] Kim C, Seo K D. Robust DTW-based recognition algorithm for hand-held consumer devices[J]. IEEE Trans-actions on Consumer Electronics, 2005, 51(2):699-709.

[5] Salvador S, Chan P. Toward accurate dynamic time warping in linear time and space[J]. Intelligent Data Analysis, 2007, 11(5):561-580.

[6] Xu H, Wang P, Liu D, et al. Maintenance Technologies Research of High Speed Turnout[J]. Applied Mechanics \& Materials, 2011, 97-98:114-120.

[7] Huang S Z, Chen W, Zhang F. et al. Method of Turnout Fault Diagnosis Based Frechet Distance[J]. Journal Of Tongji University, 2018,46(12):1690-1694.

\section{Acknowledgments}

This work was partly supported by the National key research and development program of China under Grant No. 2018YFB120500, the National Natural Science Foundation of China under Grant No. 61773313, No. 61702411. 